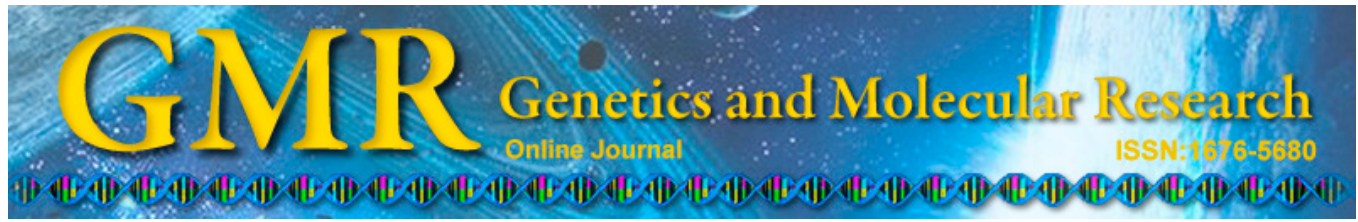

\title{
Identification and analysis of the TIFY gene family in Gossypium raimondii
}

\author{
D.H. He ${ }^{1 *}$, Z.P. Lei ${ }^{2 *}$, B.S. Tang ${ }^{1}$, H.Y. Xing ${ }^{1}$, J.X. Zhao ${ }^{1}$ and Y.L. Jing ${ }^{1}$ \\ ${ }^{1}$ College of Agronomy, Northwest A\&F University, Yangling, Shaanxi, China \\ ${ }^{2}$ College of Life Sciences, Northwest A\&F University, Yangling, Shaanxi, China \\ *These authors contributed equally to this study. \\ Corresponding author: D.H. He \\ E-mail: daohuahe@nwafu.edu.cn
}

Genet. Mol. Res. 14 (3): 10119-10138 (2015)

Received December 8, 2014

Accepted May 4, 2015

Published August 21, 2015

DOI http://dx.doi.org/10.4238/2015.August.21.19

\begin{abstract}
The highly conserved TIFY domain is included in the TIFY protein family of transcription factors, which is important in plant development. Here, 28 TIFY family genes were identified in the Gossypium raimondii genome and classified into $J A Z$ (15 genes), ZML (8), PPD (3), and TIFY (2). The normal (TIF[F/Y]XG) motif was dominant in the TIFY family, excluding the ZML subfamily, in which TLSFXG was prevalent. TIFY family genes were unevenly distributed in the G. raimondii genome, with TIFY clusters present on chromosome 9. Phylogenetic analysis indicated abundant variations in the G. raimondii TIFY family, which were most closely related to those in Theobroma cacao among 5 species. Exon-intron organization and intron phases were homologous within each subfamily, correlating with their phylogeny. Intra-species synteny analyses indicated that genomic duplication contributed to the expansion of the TIFY family. Inter-species synteny analyses indicated that synteny regions involved in $G$. raimondii TIFY family genes were also present in the comparison of G. raimondii vs Arabidopsis thaliana or T. cacao, signifying that these genes had common ancestors and play the same or similar roles
\end{abstract}


in biological processes. Greater synteny was present in the comparison of $G$. raimondii vs $T$. cacao than of $G$. raimondii vs $A$. thaliana. The expression patterns of TIFY family genes were characterized and most TIFY family genes were indicated to be involved in fiber development. Our study provides new data related to the evolution of TIFYs and their role as important regulators of transcription; these data can be useful for fiber development.

Key words: Biological evolution; Gossypium raimondii; Phylogeny; Sequence analysis; Synteny; TIFY gene family

\section{INTRODUCTION}

Transcription factors (TFs), a class of proteins that exists in all cells, govern target gene transcription by binding to DNA near target genes (Wray et al., 2003). By interacting with some transcriptional regulators such as chromatin remodeling and modifying proteins, TFs facilitate or hinder RNA polymerase from approaching the promoters of target genes, hence activating or repressing expression. In plants, transcriptional regulation plays pivotal roles in developmental processes and responses to environmental stress (Libault et al., 2009).

The TIFY gene family is present only in plants and is functionally annotated as TFs. TIFY proteins possess a conserved and entire TIFY domain, which is characterized by an approximately 36 -amino acid peptide and a highly conserved motif $[\mathrm{TIF}[\mathrm{F} / \mathrm{Y}] \mathrm{XG}$ has variant forms, although a specific glycine is always conserved (Vanholme et al., 2007)] within this peptide. The TIFY domain is intimately involved in both hetero- and homomeric interactions between the TIFY proteins and other specific TFs (Chini et al., 2009). Several studies have indicated that TIFY proteins are involved in the control of various biological pathways in plants, including development and the response to various phytohormones interrelated with stress.

The TIFY family was previously known as zinc-finger protein expressed in inflorescence meristem (ZIM) (Nishii et al., 2000). TIFY can be divided into 4 subfamilies (JAZ, PPD, TIFY, and ZML) depending on the diverse domain architecture in their protein sequences (Vanholme et al., 2007; Bai et al., 2011). Subfamily TIFY contains only the TIFY domain. In addition to the TIFY domain, the subfamily jasmonate (JA) ZIM-domain (JAZ) bears a C-terminal Jas domain of 27 amino acids, which possesses the unique motif $\mathrm{SLX}_{2} \mathrm{FX}_{2} \mathrm{KRX}_{2} \mathrm{RX}_{5} \mathrm{PY}$ (Staswick, 2008). Along with the TIFY domain, the subfamily ZML (including ZIM) is equipped with both a CCT domain (i.e., CONSTANS, CONSTANS-like, and TOC1) and a C2C2-GATA zinc-finger DNA-binding domain. Notably, the CCT domain N-terminal portion is similar in sequence to the Jas domain mentioned above. In the subfamily PPD, in addition to the TIFY domain with a motif TIFYSGK, it contains both a truncated Jas domain (lacking the conserved PY residue at its C-terminus) and a unique N-terminal PPD domain (Chung et al., 2009).

Currently, the TIFY family has been systemically analyzed in several plant species. The TIFY family includes 34 genes in soybean, 25 in poplar, 20 in rice, 18 in Arabidopsis, and 16 in grape (Vanholme et al., 2007; Ye et al., 2009; Zhu et al., 2013). Bai et al. (2011) conducted a systemic and exhaustive search of 14 genomes (not including Gossypium raimondii) in order to better determine the evolutionary history of TIFY proteins.

Information regarding the functions of TIFY family genes has been accumulating, particularly for Arabidopsis. Shikata et al. (2004) found that over expression of the AtZIM gene 
led to petiole and hypocotyl elongation because of increased cell elongation. White (2006) found that the deletion of the AtPPD1 or AtPPD2 gene increased lamina size, the curvature of dome-shaped (instead of flat) of leaf, and number of stomata. The JAZ subfamily was found to include repressors (rather than TFs) of transcription related to JA by 3 independent groups (Thines et al., 2007). When plant cells contain bioactive JA at low levels, JAZs bind to and repress MYC2, which belongs to positive basic helix-loop-helix (bHLH) TFs, to prevent the transcription of JA-responsive genes. Both the process of development and stimuli can induce cells to synthesize and accumulate bioactive JA, which subsequently triggers degradation of JAZ proteins. Next, JAZ-mediated repression is relieved and then bioactive (de-repressed) MYC2 promotes the transcription of JA-responsive genes. Interestingly, to avoid exhaustive degradation of JAZ repressors and replenish the JAZ protein pool, bioactive MYC2 induces JAZ gene expression. Tissue development and stimuli such as drought, high salinity, and low temperature, can initiate the expression of $J A Z$ genes and JA-responsive genes (Ye et al., 2009; Chacón-López et al., 2011; Seo et al., 2011; Zhu et al., 2013). In contrast, in healthy plant tissue, the expression of $J A Z$ genes is also involved in regulating a diverse array of important developmental progresses, such as seed germination, root growth, and flower development (Wasternack, 2007). However, in these pathways and responses, $J A Z$ expression is differentially regulated and induced to fine-tune the downstream JA-responsive genes (Demianski et al., 2012).

Cotton is the most important fiber crop worldwide. Fiber quality and productivity are affected by the reproductive developmental procedure of epidermal cells of cotton ovules. Tetraploid cultivated cottons originated from an ancient hybridization involving the ancestor of G. raimondii, which provided the $\mathrm{D}$ genome. Many studies have shown that the $\mathrm{D}$-subgenome contributes more phenotypical variability to $\mathrm{A}_{t} \mathrm{~A}_{\mathrm{t}} \mathrm{D}_{\mathrm{t}} \mathrm{D}_{\mathrm{t}}$ tetraploids than does the A-subgenome (He et al., 2007). In addition, most quantitative trait loci controlling fiber quality were mapped on the D-subgenome (Jiang et al., 1998). Thus, some genes, particularly $T F \mathrm{~s}$, on the D-subgenome are thought to be involved in fiber development. However, very little information is available regarding the TIFY gene family in cotton because Gossypium genomics data are lacking. The G. raimondii genome was recently sequenced and is publicly available (Paterson et al., 2012; Wang et al., 2012a), enabling identification and analysis of the entire set of TIFY family genes in G. raimondii.

Because the plant-specific TIFY family has important functions in transcription regulation, we conducted a comprehensive survey of and characterized the TIFY family genes in $G$. raimondii. We identified 2 TIFY, $15 \mathrm{JAZ}, 8 \mathrm{ZML}$, and 3 PPD genes. Moreover, syntenic and phylogenetic analyses revealed that both tandem and segmental duplication events contributed to the evolution of TIFY family genes in G. raimondii. To obtain insights into the involvement of $T I F Y$ genes in fiber development, we further characterized the expression pattern of $T I F Y$ family genes by mining the RNA-seq gene expression datasets, which are publicly available. Our results provide background data for further characterization of the evolution and function of TIFY genes within the genus Gossypium as well as contribute information to the future improvement of fiber yield and fiber quality by manipulating the expression of TIFY family genes.

\section{MATERIAL AND METHODS}

\section{Identification of TIFY family genes in G. raimondii}

The G. raimondii genome release (2.1) was downloaded from the website ftp://ftp. jgi-psf.org/pub/compgen/phytozome/v9.0/Graimondii/ to construct a local database. Multi- 
ple protein sequence alignment of conserved domains, such as pfam06200 (TIFY domain), pfam06203 (CCT motif), pfam09425 (CCT_2 domain, Jas), and pfam00320 (ZML), were acquired from NCBI (http://www.ncbi.nlm.nih.gov/cdd). Next, position-specific scoring matrix and hidden Markov model (HMM) profiles were built using the stand-alone version BLAST 2.2.26 release (ftp://ncbi.nlm.nih.gov/blast/executables/) and HMMER 3.0 (http://hmmer. janelia.org/). Searches of the position-specific scoring matrix and HMM profiles against the G. raimondii protein sequence database constructed based on the annotation details of the G. raimondii genome were conducted to explore TIFY domain-containing proteins using the RPSBLAST and hmmsearch programs. Against G. raimondii nucleotide sequence database, we searched for TIFY domain-encoding DNA sequences using RPSTBLASTN to determine whether any sequences were missed due to incomplete or erroneous annotation. Transcript data (see below) from RNA-sequencing were mined to examine and confirm the TIFY family genes encoding TIFY domain-containing proteins.

Using the program ps_scan.pl (ftp://ftp.expasy.org/databases/prosite), protein motifs of the putative G. raimondii TIFYs were then double-checked using PS51320 (TIFY domain profile), PS51017 (CCT domain profile), PS50114, and PS00344 (GATA-type zinc finger domain profile) to confirm the domains of the TIFY protein family.

Based on the absence or presence of the TIFY, CCT, Jas, or ZML domains in deduced amino acid sequences, the identified TIFY family genes were then classified into 4 subfamilies and named. To examine the conservation level of the TIFY and Jas functional domains in the G. raimondii TIFY protein family, we created sequence logos using the WebLogo program (Crooks et al., 2004).

\section{Phylogenetic analysis}

The multiple sequence alignments were generated using the ClustalW program (Chenna et al., 2003) with the Gonnet protein weight matrix. The gap opening/extension penalty was $10 / 0.1$ for pairwise alignment and 10/0.5 for multiple alignment. Alignments were visualized using BioEdit V 7.1.3.0 and adjusted manually according to domain sequences. Using the MEGA 5.1 software (Tamura et al., 2011), evolutionary trees were inferred using the neighbor-joining algorithm and tested using the bootstrap method (1000 replicates). Next, protein sequences of $T I F Y \mathrm{~s}$ were collected from 5 other species: Arabidopsis (Vanholme et al., 2007), rice (Ye et al., 2009), grape (Zhang et al., 2012), poplar (Bai et al., 2011), and Theobroma cacao; we constructed 4 expanded phylogenetic trees for 4 subfamilies.

\section{Exon-intron organization and sequence repeat analysis}

The est2genome program (Rice et al., 2000) was used to align the coding sequences and the corresponding genomic sequences to determine the organization of exon-introns in the G. raimondii TIFY family genes. The online FancyGene program (Rambaldi and Ciccarelli, 2009) was used to visualize the exon-intron organization and locations of the domains in protein sequences. To explore whether interspersed sequence repeats and low complexity DNA sequences were included in the TIFY family genes, the sequences of TIFY family genes, including flanking sequences obtained by extending 3000 base pairs upstream and downstream, were checked using RepeatMasker (http://www.repeatmasker.org). 


\section{Large-scale duplication and synteny analysis of genomic region containing TIFY family genes}

Tandem duplications (adjacent paralogous genes, without any intervening gene) and interspersed duplications of TIFYs were determined based on their positions on individual chromosomes. First, to explore paralogy/homology, protein-encoding genes from the G. raimondii genome were compared against the G. raimondii and other genomes (including Arabidopsis and T. cacao) using BLASTP (Wang et al., 2012b). Next, to identify putative paralogy/ homologous chromosomal regions containing TIFY family genes, syntenic regions within the G. raimondii, between G. raimondii/Arabidopsis and between G. raimondii/T. cacao genomes were detected using the MCScanX program (Tang et al., 2008; Wang et al., 2012b). Additionally, the genomic region containing close tandem TIFYS in G. raimondii was selected for comprehensive inter-species synteny analyses.

\section{Expression pattern analysis of $T I F Y$ family genes}

To preliminarily explore G. raimondii TIFY family genes, the dataset from 3 RNAseq experiments containing 17 runs were examined [from NCBI SRA databases, (Flagel et al., 2012)] to determine expression patterns (Table S1). Following curation, 10 comparisons were made between different developmental stages and between different tissues. Per the gff3 document (annotation information), genomic sequences of TIFY family genes were extracted and used as references, to which reads were aligned and mapped using the program TopHat (Trapnell et al., 2012). After mapping and assembly, Cuffdiff was used to identify differential expression of $T I F Y$ family genes across different developmental stages and in different tissues. The heatmap of expression profiles was produced using pheatmap in the R program.

\section{RESULTS}

\section{G. raimondii TIFY protein families}

Searches of the position-specific scoring matrix and HMM profiles against the $G$. raimondii nucleotide and protein sequence database resulted in the identification of 28 TIFY gene sequences encoding TIFY proteins (Table 1). To designate each protein member, the root symbol (i.e., subfamily designation) was followed by a sequential number. The proposed nomenclature for G. raimondii TIFY family genes, together with identifiers in $\sim 8 \times \mathrm{V} 2.10$ annotation (Paterson et al., 2012) and protein length, and high-scoring segment pairs in the annotation described by Wang et al. (2012a), are listed in Table 1. All subfamilies of G. raimondii TIFY contain more than 1 gene [GrJAZ (15 genes), GrZML(8), GrPPD (3), and GrTIFY(2)].

We compared the number of $G$. raimondii TIFY family genes in each subfamily with that in other available plant genomes (Table 2). Among 4 subfamilies, $J A Z$ was the largest and most abundant subfamily in each species, with $15 \mathrm{JAZ}$ genes in G. raimondii, 23 in maize, 20 in Glycine max, and 9 in cacao. Additionally, the $Z M L$ subfamily was the $2^{\text {nd }}$ most abundant. These 2 cases were consistent with the results in most other higher plants (Bai et al., 2011), such as Arabidopsis, rice, poplar, and cacao. The PPD subfamily underwent a species-specific expansion in G. raimondii, which was also observed in Selaginella moellendorffii. 
Table 1. Gossypium raimondii TIFY family genes.

\begin{tabular}{|c|c|c|c|c|c|c|c|}
\hline Gene name & Phytozome ID & Exon $^{\#}$ & $\mathrm{AA}^{\#}$ & TIFY motif & Coordinates & HSP gene $^{1}$ & $\mathrm{DP}^{2}$ \\
\hline GrJAZ01 & Gorai01G018800 & 5 & 228 & TIFYGG & Chr01: $1752256 \ldots 1754823$ & 10015133 & $\mathrm{SC}$ \\
\hline GrJAZ02 & Gorai02G021800 & 6 & 190 & TIFYNG & Chr02: $1523101 \ldots 1526607$ & - & $\mathrm{SC}$ \\
\hline GrJAZ03 & Gorai02G173700 & 7 & 362 & TIFYAG & Chr02: 44407878...44410995 & 10019921 & $\mathrm{SC}$ \\
\hline GrJAZ04 & Gorai04G285100 & 4 & 241 & TIFYCG & Chr04: 61612558...61614463 & 10010230 & $\mathrm{SC}$ \\
\hline GrJAZ05 & Gorai05G196200 & 6 & 197 & TIFYNG & Chr05: 56877532...56879768 & 10001798 & $\mathrm{SC}$ \\
\hline GrJAZ06 & Gorai06G092400 & 3 & 125 & TIFYNG & Chr06: $32947665 \ldots 32949260$ & - & $\mathrm{SC}$ \\
\hline GrJAZ07 & Gorai08G291000 & 7 & 371 & TIFYAG & Chr08: $56545806 \ldots 56551262$ & 10030605 & $\mathrm{~S}$ \\
\hline GrJAZ08 & Gorai09G036800 & 4 & 240 & TIFYGG & Chr09: $2718340 \ldots 2720664$ & 10037314 & $\mathrm{SC}$ \\
\hline GrJAZ09 & Gorai09G039500 & 5 & 226 & TIFYDG & Chr09: 2919762...2922783 & 10037287 & $\mathrm{SC}$ \\
\hline GrJAZ10 & Gorai09G145400 & 4 & 226 & TIFFGG & Chr09: $11017453 \ldots 11020374$ & 10031747 & $\mathrm{SC}$ \\
\hline GrJAZ11 & Gorai09G154300 & 3 & 119 & TIFYNG & Chr09: $11795808 \ldots 11797204$ & 10033602 & SC \\
\hline GrJAZ12 & Gorai09G330500 & 7 & 365 & TIFYAG & Chr09: $34149627 \ldots 34152849$ & 10005893 & $\mathrm{SC}$ \\
\hline GrJAZ13 & Gorail0G090600 & 4 & 263 & TIFYGG & Chr10: $13926370 \ldots 13929049$ & 10039035 & $\mathrm{SC}$ \\
\hline GrJAZ14 & Gorai11G045300 & 3 & 120 & TIFYNG & Chr11: 3453543...3454792 & 10031542 & $\mathrm{SC}$ \\
\hline GrJAZ15 & Gorail1G062000 & 5 & 270 & TIFFGG & Chr11: 5133243...5135195 & 10023394 & $\mathrm{SC}$ \\
\hline GrZML1 & Gorai02G138300 & 9 & 356 & TLSFEG & Chr02: $23533661 \ldots 23537596$ & 10038826 & $\mathrm{SC}$ \\
\hline GrZML2 & Gorai05G097100 & 7 & 285 & TLSFRG & Chr05: $14683753 \ldots 14688216$ & 10039389 & $\mathrm{SC}$ \\
\hline GrZML3 & Gorai05G226000 & 11 & 390 & TIAFEG & Chr05: $60845139 \ldots 60850463$ & 10000634 & $\mathrm{SC}$ \\
\hline GrZML4 & Gorai08G017800 & 7 & 314 & TLSFQG & Chr08: $1998002 \ldots 2001547$ & 10038217 & $\mathrm{SC}$ \\
\hline GrZML5 & Gorai09G279200 & 5 & 282 & TLSFRG & Chr09: 23544503...23549391 & 10023684 & $\mathrm{SC}$ \\
\hline GrZML6 & Gorai10G033400 & 11 & 360 & TLSFEG & Chr10: $3006376 \ldots 3011496$ & 10011724 & $\mathrm{SC}$ \\
\hline GrZML7 & Gorai10G033500 & 7 & 296 & TLSFRG & Chr10: $3023264 \ldots 3027641$ & 10011723 & NS \\
\hline GrZML8 & Gorai13G055000 & 7 & 314 & TLSFQG & Chr13: 5393873...5397619 & 10029545 & $\mathrm{SC}$ \\
\hline GrPPDI & Gorai04G077100 & 9 & 345 & TIFYCGK & Chr04: $8931782 \ldots 8937389$ & 10020204 & SC \\
\hline GrPPD2 & Gorai08G002400 & 9 & 341 & TIFYCGK & Chr08: 410942...416444 & 10038066 & $\mathrm{SC}$ \\
\hline GrPPD3 & Gorai11G168900 & 9 & 365 & TIFYCGK & Chr11: $34616085 \ldots 34621616$ & 10031607 & $\mathrm{~S}$ \\
\hline GrTIFY1 & Gorai02G216400 & 3 & 140 & TIFYAG & Chr02: $56617136 \ldots 56618433$ & 10004804 & $\mathrm{~S}$ \\
\hline GrTIFY2 & Gorai09G011200 & 6 & 427 & TIFYGG & Chr09: $903341 \ldots 907617$ & 10016076 & $\mathrm{~S}$ \\
\hline
\end{tabular}

${ }^{1} \mathrm{HSP}$ : High-scoring segment pairs (HSPs) show the alignments of the query and hit sequence from gene annotations of Wang et al. (2012a). Cotton_D_gene_10015133 was abbreviated to 10015133. ${ }^{2}$ DP, Duplication; SC, located in syntenic (duplicated) genome region, and has the syntenic TIFY family counterpart; S: shown in syntenic regions, the syntenic TIFY family counterpart was lost; NS, not mapped within any synteny blocks.

Table 2. TIFY family genes found in genomes of several plants including Gossypium raimondii.

\begin{tabular}{lcccccccccrrrrrrrrr}
\hline Subfamily & $\mathrm{Ol}$ & $\mathrm{Ot}$ & $\mathrm{Pp}$ & $\mathrm{Sm}$ & $\mathrm{Mg}$ & $\mathrm{At}$ & $\mathrm{Pt}$ & $\mathrm{Vv}$ & $\mathrm{Gm}$ & $\mathrm{Gs}$ & $\mathrm{Pv}$ & $\mathrm{Mt}$ & $\mathrm{Gr}$ & $\mathrm{Tc}$ & $\mathrm{Os}$ & $\mathrm{Zm}$ & $\mathrm{Sb}$ & $\mathrm{Bd}$ \\
\hline JAZ & 0 & 0 & 9 & 8 & 9 & 12 & 12 & 7 & 20 & 18 & 11 & 14 & 15 & 9 & 15 & 23 & 16 & 15 \\
ZML & 0 & 0 & 4 & 3 & 1 & 3 & 8 & 4 & 9 & 9 & 4 & 5 & 8 & 3 & 4 & 3 & 3 & 6 \\
PPD & 0 & 0 & 0 & 4 & 2 & 2 & 2 & 2 & 2 & 1 & 1 & 0 & 3 & 2 & 0 & 0 & 0 & 0 \\
TIFY & 0 & 0 & 3 & 2 & 1 & 1 & 3 & 2 & 3 & 6 & 3 & 2 & 2 & 1 & 1 & 1 & 0 & 0 \\
Reference & $\mathrm{A}$ & $\mathrm{A}$ & $\mathrm{B}$ & $\mathrm{B}$ & $\mathrm{B}$ & $\mathrm{B}$ & $\mathrm{B}$ & $\mathrm{B}$ & $\mathrm{B}$ & $\mathrm{C}$ & $\mathrm{D}$ & $\mathrm{B}$ & - & - & $\mathrm{B}$ & $\mathrm{B}$ & $\mathrm{B}$ & $\mathrm{B}$ \\
\hline
\end{tabular}

At, Arabidopsis thaliana; Bd, Brachypodium distachyon; Gm, Glycine max; Gr, Gossypium raimondii; Gs, Glycine soja; Mg, Mimulus guttatus; Mt, Medicago trunculata; Ol, Ostreococcus lucimarinus; Os, Oryza sativa; Ot, Ostreococcus tauri; Pp, Physcomitrella patens; Pt, Populus trichocarpa; Pv, Phaseolus vulgaris; Sm, Selaginella moellendorffi; $\mathrm{Sb}$, Sorghum bicolor; Tc, Theobroma cacao; $\mathrm{Vv}$, Vitis vinifera; Zm, Zea Mays. Numbers of the genes in all plants (excluding Gr and Tc) are obtained from literature. A, Vanholme et al. (2007); B, Bai et al. (2011); C, Zhu et al. (2013); D, Aparicio-Fabre Rosaura et al. (2013).

HMMER 3.0 analysis was also conducted on the G. raimondii genome sequence released by Wang et al. (2012a) and 29 genes were found to belong to the TIFY gene family. The most homologous proteins from Paterson et al. (2012) are listed in Table 1 based on BLASTp. Two genes (GrJAZ02 and GrJAZ06) had no explicit counterpart in gene annotation with the results of Wang et al. (2012a). Except for those listed in Table 1 (in the $7^{\text {th }}$ column), 3 genes (Cotton_D_ gene_10001798, 10031542, and 10038825) were found to belong to the TIFY family. 
Multiple sequence alignment and the logo of the TIFY domain indicated that entire TIFY domain had diverse forms, but was conserved in the motif (TIF[F/Y]XG, Figure 1).

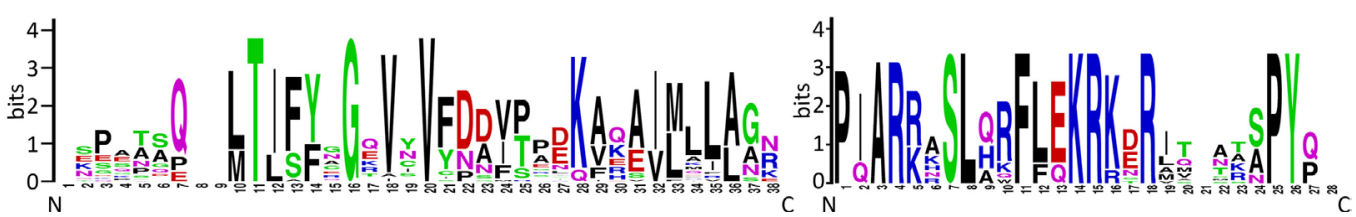

Figure 1. Sequence logo of TIFY (left) and Jas (right) domains in the family TIFY proteins from Gossypium raimondii.

Moreover, the TIFY motifs (only for the ZML subfamily) deviated from the typical motif (TIF[F/Y]XG) and included TLSFXG (7 proteins) and TIAFEG (1). These results indicate that TIF[F/Y]XG is dominant in the TIFY family [excluding the ZML subfamily, in which TLSFXG is dominant (Bai et al., 2011)]. In subfamily PPD, the TIFY domain contains a Gossypium-specific TIFYCGK rather than a general TIFYSGK (Chung et al., 2009) motif. A few amino acids are included in the consensus and thus were present in all of the G. raimondii TIFY proteins identified. In contrast, the logo of the Jas motif in G. raimondii showed a higher level of conservation. Particularly, at 11 sites $(1,3,4,7,8,11,14,15,18,25$, and 26), the amino acids observed were conserved. Additionally, conserved amino acid sequences of RX(2-5)PY were located at the C-terminal end of the Jas domain. This conserved region was thought to be a nuclear location signal and JAZ is located in the nucleus in vivo (Grunewald et al., 2009). The logo of the GATA zinc finger domain (data not shown) indicated that among 37 amino acids, 26 were highly conserved. At 29 of 45 sites in the CCT motif, amino acid residues were also strongly conserved, showing $100 \%$ identity.

\section{Phylogenetic analysis of TIFYs in G. raimondii and additional 5 species}

Amino acids in the TIFY family were diverse, even in the peptide region of the TIFY domain, in which the hydrophobic amino acids were more variable (Vanholme et al., 2007). Additionally, the number of amino acids in the G. raimondii TIFY protein sequences varied from 119-427 (Table 1). Therefore, phylogenetic analysis of the 28 G. raimondii TIFY members was performed according to the alignments of full-length protein sequences, which were adjusted exclusively according to conserved domain sequences to increase reliability. Figure 2 displays the G. raimondii TIFY family tree.

The phylogenetic tree illustrated that G. raimondii TIFY family genes were classified into 9 clades (marked by 9 red dots in Figure 2). JAZs and ZMLs were subdivided into 5 and 2 distinct clades, respectively. Clades 3 and 6 were the largest clades, with each including 5 genes. Clades 7 and 9 showed relatively low sequence identity. Members in different clades show great divergence in sequence, but those in the same clade were closely related. Evidence includes that proteins clustering together generally contained approximately the same number of amino acids. Additionally, TIFY family proteins from the same subfamilies appeared to be clustered together. Genes from subfamily TIFY diverged significantly from the homologs in the other 3 subfamilies, and were phylogenetically the most distantly related subfamilies.

To obtain information related to the functional relevance of abundant TIFY family members in G. raimondii, the phylogenetic relationships of the proteins in each subfamily were further analyzed between $G$. raimondii TIFYs and other TIFY family members from 
5 species, including Arabidopsis, rice, poplar, cacao, and grape. The expanded phylogenetic trees for each subfamily were constructed (Figure 3).

The tree produced from the phylogenetic analyses of 6 species indicated that all members of the TIFY families in G. raimondii were most closely related to those in cacao compared to those in other species, and showed the lowest relationship with rice. The similarity coefficient between protein sequences were then checked and the results indicated that most $(26 / 28=92.86 \%)$ G. raimondii TIFY members were more closely related to those in $T$. cacao (average of identity $=0.1857$ for GrJAZ vs TcJAZ, 0.4929 for GrZML $v s$ TcZML, and 0.5645 for GrPPD $v s$ TcPPD), while the minority $(2 / 28=7.14 \%)$ were more closely related to those in Populus trichocarpa.

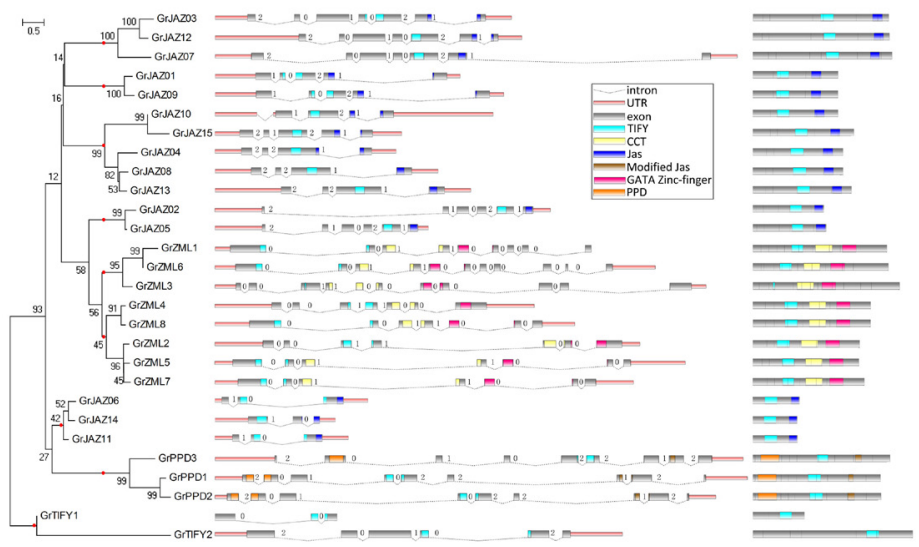

Figure 2. Phylogenetic relationship (left), exon-intron organizations (middle), and the distribution of conserved domains in proteins (right) of Gossypium raimondii TIFYs. Phylogenetic analysis was performed using the neighbor-joining method. A phylogram was created using TIFY protein sequences and ClustalW2 multiple sequence alignment software. The tree was produced using the MEGA5.1 software. The numbers shown above or below tree branches show bootstrap values. Red dots on branches indicate 9 clades. Exon-intron organizations of TIFY family genes were drawn using FancyGene (http://bio.ieo.eu/fancygene/). The intron phase (number on intron) shows the intron location in a codon. Phase 0 indicate introns between 2 codons, while phase 1 shows introns between the 1 st and 2 nd bases of a codon. Phase 2 shows introns between the 2 nd and $3 \mathrm{rd}$ bases. Colors indicate each conserved domains with relative position within each protein.

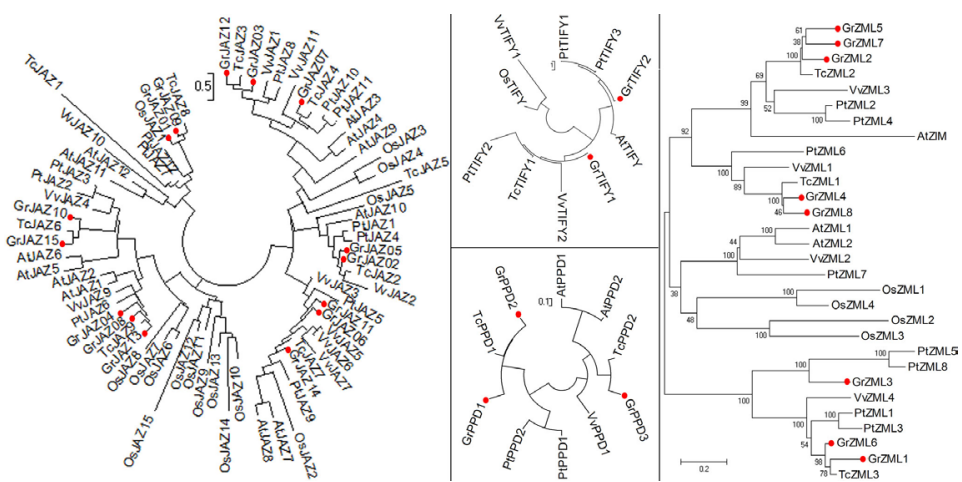

Figure 3. Phylogenetic analysis of sequences of TIFY proteins (4 subfamilies) from 6 species including Gossypium raimondii (Gr), Arabidopsis thaliana (At), Oryza sativa (Os), Populus trichocarpa (Pt), Theobroma cacao (Tc), and Vitis vinifera $(\mathrm{Vv})$. 


\section{Exon-intron organization and sequence repeat analysis of $T I F Y$ family genes}

The divergence of the exon-intron organization within the TIFY family played a critical role in the evolution of multiple gene families, and thereafter, phylogenetic groupings and evolutionary relationships were further supported (Shiu and Bleecker, 2003). To obtain further insights into structural evolution, the exon-intron organization in the TIFY family genes were investigated. Our results revealed that all members of the TIFY family possessed from 2-10 introns, and the most closely related genes within the same clade (even subfamilies) generally featured similar exon-intron organization in terms of either intron numbers or exon lengths (Figure 2), correlating the phylogeny illustrated by the tree. In the same clade, genes exhibited nearly identical exon lengths and intron numbers, a relatively constant exon-intron composition, and significantly different intron lengths. However, there were striking distinctions in the arrangement of introns among clades, supporting the results of phylogenetic analysis. These results, along with the moderate sequence identity, suggest that G. raimondii TIFY family members may have undergone gene differentiation after independent gene duplications throughout evolution to generate paralogs, resulting in the TIFY family featuring diverse exonintron organization. The length ratio of the coding sequence $v s$ the transcript region ranged from $16.2 \%$ (GrJAZ02) to $63.7 \%$ (GrJAZ13).

All G. raimondii PPD genes possessed a similar number (up to 8 ) of introns in their coding sequences, which was the same as that of AtPPD in Arabidopsis (Bai et al., 2011). However, the gene structures in G. raimondii JAZ subfamilies appeared to be more variable, displaying abundant variants in exon-intron organization, i.e., G. raimondii JAZ possessed 2-6 introns. Additionally, the most phylogenetically divergent JAZ genes (GrJAZ06, GrJAZ11, and GrJAZ14) displayed an exon-intron organization that was dissimilar to that of the other GrJAZ genes. For G. raimondii $Z M L$, the number of introns in 8 members varied from 6-10. GrZML1 was not identical to other GrZML genes with 6 or 8 introns. Overall, most $Z M L$ members contained 6 introns in their coding regions, which is the same as in grape $Z M L$ genes (Zhang et al., 2012). The gene structures of the GrJAZ subfamily were more divergent than those of the GrZML subfamily. Based on the exon-intron organization, the GrJAZ and GrZML (except GrZML1) genes were divided into 5 and 2 subgroups, respectively. Two GrTIFY genes were quite different in exon-intron organization, and perhaps only 1 conserved domain was present in the TIFY subfamily, which had more variability in its sequence. Furthermore, comparison of the exon-intron organization of TIFY family genes among several species, including G. raimondii, Arabidopsis, cacao, and grape, indicated that the exon-intron organizations of each subfamily were analogous across these 4 species (data not shown).

The relative positions and distribution of each conserved domain were constant within each protein sequence in the TIFY family. For some genes, introns were embedded in the genomic sequence region encoding conserved domain such as the TIFY domain and PPD domain. Particularly, GrJAZ10 contained 1 intron in the 5' untranslated region. One intron was generally present between the TIFY domain and CCT_2 (Jas) domain-encoding sequence. CCT_2 was generally present in the final downstream exon, except GrJAZ07, which featured an unusually long intron in the $3^{\prime}$ region and no intron embedded in the sequence encoding the CCT_2 domain. One intron was generally present between the CCT and GATA domainencoding sequence, except for GrZML2 and GrZML8. In the PPD subfamily, 2 introns were present in the region between sequences encoding the TIFY domain and modified Jas, and 2 other introns were present (nested) in the region between sequences encoding the PPD and 
TIFY domains, except GrPPD3 which contained a 3rd intron in that region.

We also investigated the intron phases with respect to codons. Eight GrZML genes showed 3 intron phase patterns, among which the difference was only in the length $(0001100$, 000110000, and 00011000000). Three GrPPD members showed an intron phase pattern (020102212). The GrJAZ genes showed 7 intron phase patterns, including a long pattern (0201021) with 3 genes (GrJAZ3, GrJAZ07, and GrJAZ12), while the remaining 6 patterns were short and appeared to be extracted from the long pattern (0201021) with conserved order. GrTIFY subfamily members had different intron phase patterns (000 and 020102 , which appeared to be extracted from that of GrJAZs or GrPPDs), similar to the long pattern of GrJAZs and GrPPDs. The intron pattern of the GrZML genes was quite different from those of the GrJAZ and GrPPD genes. This supported that GrZMLs vs GrPPDs subfamilies were less related than GrJAZs vs GrPPDs.

An intron was inserted in the TIFY domain of 17 TIFY family genes, and most (14/17) were the phase 0 intron. Phase 0 and 1 introns were embedded within sections encoding the CCT domain of 3 and 6 GrZML genes, respectively. An intron was inserted in the Jas domain of 8 GrJAZ genes, and most (7/8) were the phase 1 intron. These results indicated a high level of conservation of the intron phase in the Jas and TIFY domains compared with other sections of TIFY family genes.

The output of the RepeatMasker program indicated that most (22/28) TIFY family genes contained simple repeats, implying that economical SSR markers can be adopted to mark and track these genes. Low-complexity sequence repeats (such A-rich and GA-rich) were present in transcribed genomic regions of 8 TIFY family members. Long terminal repeat retrotransposons (such as gypsy and copia) and long interspersed nuclear elements (such as L1) were embedded in the vicinity of 5 and 4 genes, respectively. Additionally, MuLE-MuDR and PIF/Harbinger were related to the up- and downstream regions of 3 and 1 genes, respectively. Sequence repeats including transposons make up more than $57 \%$ of the DNA in $G$. raimondii (Wang et al., 2012a).

\section{Chromosomal distribution of $T I F Y$ family genes and duplication regions in the $G$. raimondii genome}

The chromosomal locations of TIFY family genes were assigned based on the G. raimondii Genome Release 2.1 at Phytozome. TIFY family genes were present on all chromosomes (Chr), except $\mathrm{Chr}$ 03, 07, and 12 (Figure 4). The location distribution of TIFY family genes was similar in G. raimondii, Arabidopsis thaliana, and Sorghum bicolor, with no less than 7 TIFY genes present on a single chromosome (Bai et al., 2011). TIFY family gene clusters were distributed on Chr 09 in G. raimondii, Chr 01 in A. thaliana, and Chr 01 in S. bicolor. Particularly, tandem duplications present on G. raimondii $\mathrm{Chr} 09$ and $\mathrm{Chr} 10$ were similar to those on $S$. bicolor $\mathrm{Chr} 01$ and Oryza sativa Chr03. In contrast, TIFY genes were generally dispersed in the soybean and grape genomes, with no more than 4 TIFY genes present on a single chromosome.

Tandem duplications typically resulted in gene family expansion (Cannon et al., 2004). In this study, close tandem duplications were found in the $Z M L$ subfamily (GrZML6/ GrZML7). Interestingly, these close tandem duplications were also found in grape [VvZML2/ VvZML3 (Zhang et al., 2012)], poplar (PtZML1/PtZML2, PtZML5/PtZML6), Medicago truncatula (MtZML1/MtZML2), G. max (GmZML2/GmZML3, GmZML4/GmZML5), and Brachypodium distachyon (BdZML4/BdZML5). These collections are referred to as close tandem 
duplications, which are defined as adjacent and paralogous genes within a chromosome without intervening genes. Additionally, GrJAZ08 and GrJAZ09 were found to be near tandem duplications, which were also found in rice (OsJAZ9/OsJAZ10/OsJAZ11), grape (VvJAZ4/ VvJAZ5), and maize (ZmJAZ5/ZmJAZ6).

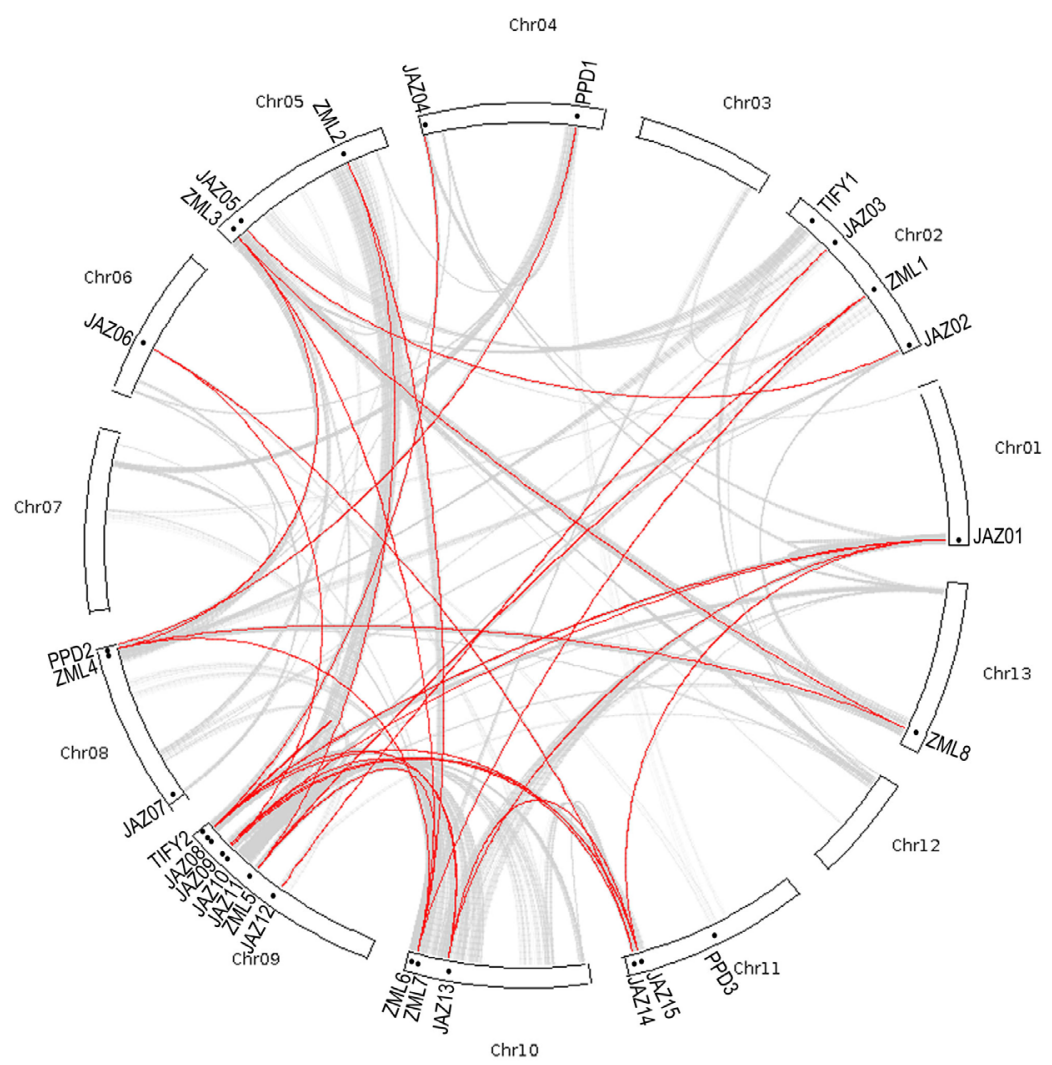

Figure 4. Distribution and synteny of TIFY family genes on Gossypium raimondii chromosomes. Chromosome (Chr) 01-13 are depicted as curve white bars. TIFY family genes are indicated by black dots. Gray curve lines denote syntenic regions containing TIFY family genes (no counterpart); red curve lines indicates syntenic regions (counterparts were present).

In addition to tandem duplications, dispersed segmental duplications were also important factors facilitating gene family expansion (Cannon et al., 2004). We then surveyed the duplicated regions within the G. raimondii genome using MCScanX and identified 23 TIFY family genes located within 79 pairs of duplicated (syntenic) blocks (Figure 4), which had a syntenic $T I F Y$ family counterpart. Therefore, most $T I F Y$ family genes may have evolved from direct genomic segmental duplications. Eight alignments (involving GrTIFY1, GrTIFY2, GrJAZ07, and GrPPD3) from synteny analysis revealed no syntenic TIFY family counterpart, possibly as a result of a lost gene or shuffle. GrZML7 was the only gene that was not mapped into any synteny blocks. In summary, 96\% (27/28) of TIFY genes were detected in synteny regions. No synteny was found among different subfamilies. 
Among large-scale segmental duplicated regions, 3 collections contained more than one $T I F Y$ per region. One collection consisted of 5 genomic blocks (forming 5 alignments), including the block of GrJAZ01-Gorai01G016500 on Chr 01, block of Gorai04G285700GrJAZ04 on Chr 04, block of GrJAZ09-GrJAZ08 on Chr 09, block of Gorail0G094100GrJAZ13 on Chr 10, and block of Gorail3G221600-Gorail3G222700 on Chr 13 (non-TIFY genes illustrated that the suppositional syntenic TIFYs counterpart should be near the loci). This collection belonged to pentaplicated regions that included 5 TIFYs. The other collection (4 alignments, tetraplicated) contained GrZML3 (on Chr 05), GrZML4 (on Chr 08), GrZML8 (on Chr 13), and Gorai12G143900 (on Chr 12). The $3^{\text {rd }}$ collection (3 alignments, triplicated) contained GrJAZ10 (on Chr 09), GrJAZ13 (on Chr 10), and GrJAZ15 (on Chr 11). Many studies have shown that during the long process of evolution, plant genomes underwent several rounds of large-scale-duplication events (Paterson et al., 2012). Subsequently, similar functional genes were partly retained. This viewpoint supports the expansion of many TF families through genomic duplication followed by rearrangement in the plant kingdom (Wang et al., 2012b). Genome-wide duplication events within Gossypium may account for the expansion of the G. raimondii TIFY family. The 3 collections supported that abundant $G$. raimondii TIFY family genes may have descended from massive genomic region expansion (such as duplication, triplication, and pentaplication) and diversification following duplication. In contrast, the genes descending from a single ancestral gene were typically included in a clade, therefore constituting a paralogous group.

After intra-species synteny analyses, we found that each gene cluster (excluding TIFY subfamily) originating from tandem or segmental duplication events generally shared similar exon-intron organizations with only minor differences. Indeed, further evaluation indicated that the TIFY family genes derived through duplication events contained exactly the same number of exons, which were similar to each other in exon length. This was supported by 4 sets of genes (GrJAZ04/GrJAZ08/GrJAZ13, GrZML2/GrZML5/GrZML7, GrJAZ06/GrJAZ11/ GrJAZ14, and GrPPD1/GrPPD2/GrPPD3).

\section{Evolutionary relationships of $T I F Y$ family genes between $G$. raimondii and other species}

The genomic sequence comparison between different taxa provided the dataset for reconstructing the evolution of each gene (Koonin, 2005) and was used to infer the characterization of genes in taxa that had not been well studied, based on the abundant data from other well-studied taxa (Lyons et al., 2008). Many functions of Arabidopsis TIFY family genes have been well studied. Therefore, we deduced the roles of the G. raimondii TIFY family from Arabidopsis homologs identified by comparative genomics. Additionally, because the protein sequences of the TIFY family in G. raimondii bear the closest relationship with that in T. cacao, we compared the genomic sequence of Arabidopsis vs G. raimondii and cacao vs G. raimondii, followed by visualization of syntenic regions containing G. raimondii TIFY family genes using MCScanX (Tang et al., 2008; Wang et al., 2012b). Eighty and 43 largescale syntenies involving GrTIFY family genes (26 and 27) were identified for Arabidopsis vs G. raimondii and cacao vs G. raimondii, respectively. However, only 16 and 23 GrTIFY family genes (involved in 25 and 30 syntenies) had counterparts in Arabidopsis and cacao, respectively (Figure 5). The biunique syntenies unambiguously indicated that the homologous gene-pairs evolved from the last common ancestor. Biunique gene-pairs (including GrJAZ03AtJAZ9, GrZML6-AtZIM, GrTIFY2-AtTIFY, GrJAZ03-TcJAZ3, and GrTIFY1-TcTIFY1) were 
scarce. Most syntenies involved many-to-one or many-to-many homologous relationships. For example, duplicated or triplicated GrTIFY genes corresponded to a single gene in Arabidopsis or cacao. The case included (GrJAZ02/GrJAZ05)-AtJAZ10, (GrPPD1/GrPPD3)-AtPPD1, and (GrJAZ06/GrJAZ11/GrJAZ14)-TcJAZ7, which supported the deduction that the common ancestral gene had expanded in G. raimondii, but not in Arabidopsis or cacao. Some ancestral genes did expand a same number of times in each species after speciation of Arabidopsis, cacao, and G. raimondii from the last common ancestor. This was supported by (GrJAZ01/GrJAZ09)(AtJAZ11/AtJAZ12) and (GrJAZ11/GrJAZ14)-(AtJAZ7/AtJAZ8). Furthermore, there were cases where multiplicated GrTIFY genes corresponded to duplicated genes [such as (GrPPDI/ GrPPD2/GrPPD3)-(TcPPD1/TcPPD2), and (GrZML1/GrZML2/GrZML4/GrZML5/GrZML6/ GrZML8/GrZML9)-(TcZML1/TcZML2)] and multiplicated GrTIFY genes corresponded to triplicated genes [such as (GrJAZ08/GrJAZ10/GrJAZ13/GrJAZ15)-(AtJAZ1/AtJAZ6/AtJAZ5), and (GrJAZ01/GrJAZ04/GrJAZ08/GrJAZ09/GrJAZ10/GrJAZ13/GrJAZ15)-(TcJAZ6/TcJAZ8/Tc$J A Z 9)]$. Therefore, an ancestral gene has expanded more times in G. raimondii but fewer times in Arabidopsis and cacao during the evolution following speciation.

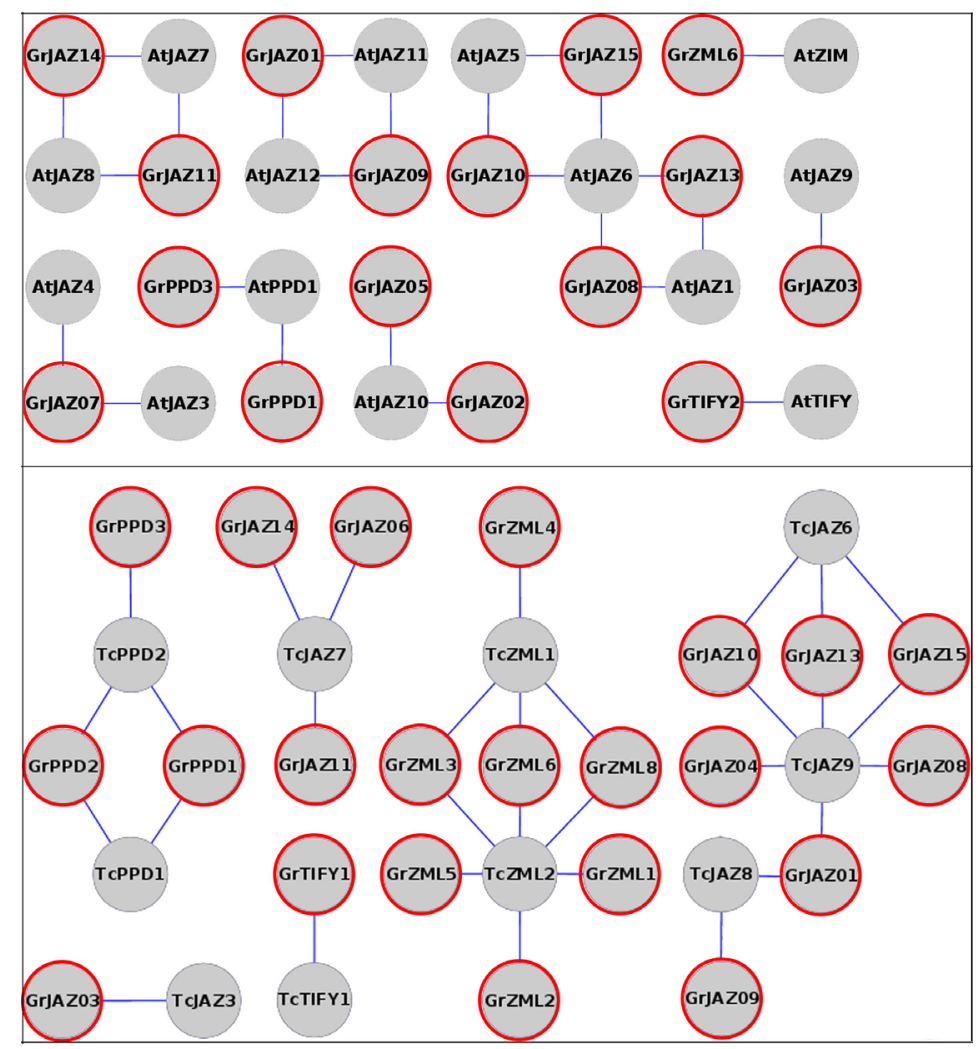

Figure 5. Network of the synteny between inter-species $T I F Y$ genes. The MCScanX software allows the detection of syntenic relationships existing between the TIFY family genes. Nodes represent genomic region containing the genes and the edges can be thought of as synteny/duplication relationships. The red nodes indicate genes of Gossypium raimondii, while other nodes indicate genes of Theobroma cacao (Tc) and Arabidopsis thaliana (At). Inter-species syntenies, which do not involve GrTIFY family genes or involve GrTIFY family genes without counterpart in other species, are not illustrated in this figure. 
Except for the GrTIFY members mentioned above that had syntenic counterparts in other species, some genes, including GrJAZ04, GrJAZ06, GrJAZ12, GrZML1, GrZML3, GrZML4, GrZML7, GrZML8, GrPPD2, and GrTIFY1, were also embedded in the syntenic regions of Arabidopsis vs G. raimondii, whereas their respective syntenic Arabidopsis counterpart had been lost. Similarly, the comparison of cacao vs G. raimondii showed that 4 genes, including GrJAZ02, GrJAZ05, GrJAZ07, and GrZML7, which were also located in the syntenic regions, lost their respective syntenic cacao counterparts. Additionally, 2 genes, GrZML2 and GrZML5, and 1 gene, GrJAZ12, had not been mapped into any synteny blocks of $G$. raimondii_Arabidopsis and G. raimondii_cacao, respectively. Perhaps, these GrTIFY members did not share a common ancestral gene with Arabidopsis or cacao. The failure of the syntenies identification may have resulted from selective gene loss, which typically followed chromosomal rearrangement (fracture and fusions) during evolution (Wang et al., 2012a) after speciation of G. raimondii, Arabidopsis, and cacao. Further studies are necessary to explain the syntenic observations.

In general, inter-species synteny identifications, involving GrTIFY members, indicated that syntenic regions of $G$. raimondii_cacao (up to 310 genes) were longer than that of G. raimondii_Arabidopsis (no more than 40 genes). Furthermore, syntenic regions of $G$. raimondii_cacao involved more GrTIFY members than those of G. raimondii_Arabidopsis. These results also implied that G. raimondii was more closely related to cacao than to Arabidopsis, in agreement with the phylogenetic analysis described above.

As described above, 2 GrZML members, GrZML6 and GrZML7, were closely tandem duplicates of each other on Chr 10. This was also observed in several species. Thus, the genomic region containing GrZML6 and GrZML7 was extracted to carry out comprehensive inter-species synteny detection. The results indicated that syntenies involving the GrZML6GrZML7 region were present in several genomic comparisons, such as P. trichocarpa_G. raimondii, G. max_G. raimondii, T. cacao_G. raimondii, V. vinifera_G. raimondii, and $M$. truncatula_G. raimondii (Table S2). We then investigated the genes in the vicinity of GrZML6 and $G r Z M L 7$ to gain additional insight into the level of conservation. The data indicated that homologs of Gorai10G033700, Gorai10G033900, and Gorai10G034400 were present in the comparisons to several species, including poplar, soybean, and cacao. Additionally, the syntenies containing the near-tandem GrJAZ08 - GrJAZ09 region were also present in 4 comparisons (3 in G.raimondii_G.max, 1 in G.raimondii_T. cacao, 2 in G.raimondii_M. truncatula, and 1 in G. raimondii_P. trichocarpa), with counterparts of GrJAZ08 and GrJAZ09 present. Attention should be given to Gorai09G036700, which always linked with GrJAZ08 among the 10 species. Several enzymes, including the TIFY family members, encoded by closely linked genes, involved in a metabolic pathway co-evolved similarly in each species.

\section{Expression profiles of $T I F Y$ family genes}

In plants, TIFY family genes play important roles in biological development and the adaptation to diverse stresses (Vanholme et al., 2007; Zhang et al., 2012). Most TIFY family genes appear to have a different spatiotemporal pattern of expression within different genera and species (Bai et al., 2011). Therefore, functional characterization of TIFY provided basic data for further improvement of plant production quantity and quality. In this study, the expression profiles of G. raimondii TIFY family genes were studied. The heatmap of expression profiles (Figure 6) showed that most genes had different and broad expression patterns across 
different spatiotemporal domains. Twenty-six of these TIFY family genes were expressed differentially in a minimum of 1 of the 3 experiments. Detailed differences are shown in Table $\underline{\mathbf{S 3}}$ and Figure S1.

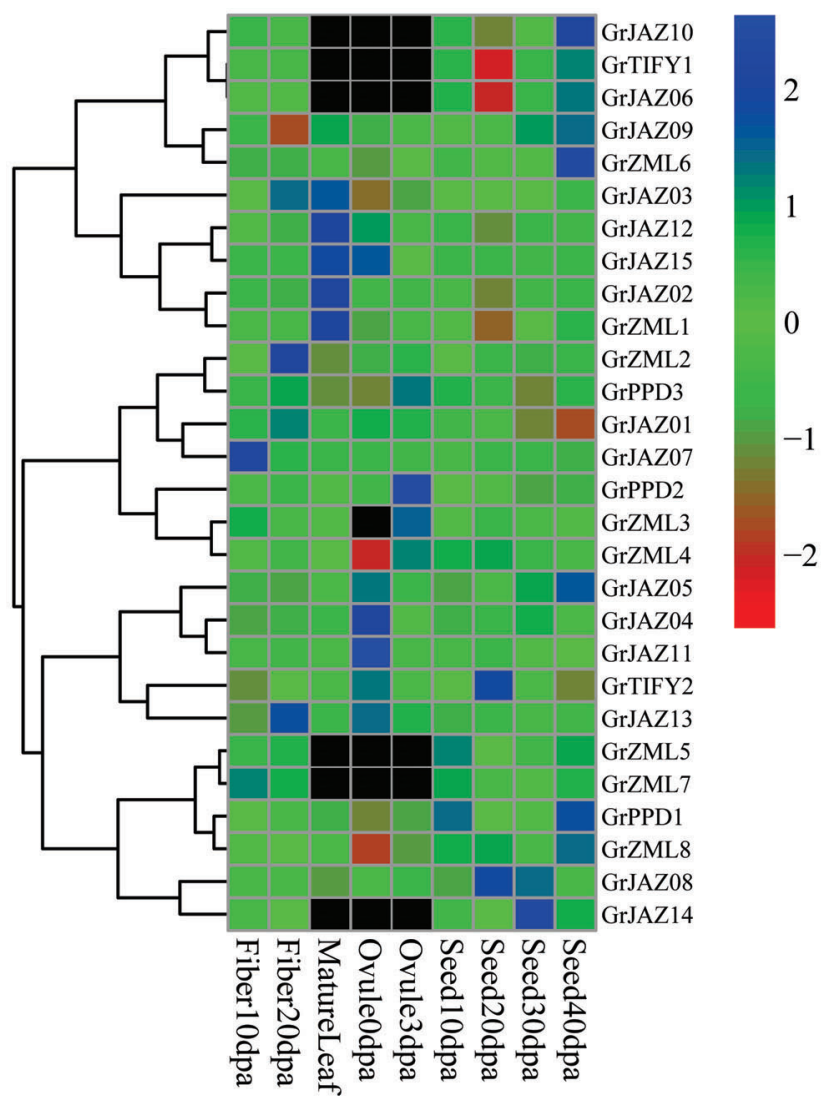

Figure 6. Expression profile of TIFY family genes. Levels of gene expression are depicted in different color on the right scale. Black: missing value.

Fiber growth is an important developmental process controlling the productivity and quality of lint, which shares many similarities with $A$. thaliana leaf trichome development (Wan et al., 2014). This process includes 4 overlapping stages: initiation [0-8 days post anthesis (dpa)], elongation (i.e., primary cell wall synthesis, 3-17 dpa), secondary cell walls synthesis (17-23 dpa), and maturation (after $20 \mathrm{dpa}$ ) (Graves and Stewart, 1988). In contrast, by interacting with some factors, JAZ proteins modulated trichome initiation (Qi et al., 2011). Thus, the expression profiles of the TIFY family were examined in different tissues (leaves vs ovules) and at different developmental stages of fibers (including 0, 3, 10, 20, 30, and $40 \mathrm{dpa}$; Table S1, Figure S1). No differences in transcriptional abundance were detected for the genes GrJAZ06 and GrTIFY1 among all stages of fiber growth. Data from no less than 2 experiments indicated that 21 genes, except GrJAZ10, GrJAZ14, GrZML1, GrZML5, and GrZML7, exhibited significant expression differences during different stages. 
Regarding the comparison of different tissues, the transcription levels of 8 genes (GrJAZ02, GrJAZ03, GrJAZ09, GrJAZ12, GrZML1, GrZML4, GrZML6, and GrZML8) in vegetative tissues (leaves) were much higher than those in reproductive tissues ( 0 and 3 dpa ovules), whereas 6 genes (GrPPD2, GrJAZ05, GrJAZ07, GrJAZ08, GrJAZ13, and GrZML2) showed the opposite pattern. Additionally, 3 genes (GrJAZ05, GrJAZ11, and GrTIFY2) showed significant changes with obscure direction in expression levels between vegetative tissues and reproductive tissues. For fiber growth, at the stage of fiber initiation ( 3 vs 0 dpa), the expression abundance of 12 genes (GrJAZ01, GrJAZ02, GrJAZ04, GrJAZ05, GrJAZ08, GrJAZ09, GrJAZ11, GrJAZ12, GrJAZ13, GrJAZ15, GrPPD1, and GrTIFY2) decreased, while the expression abundance of 3 genes (GrJAZ07, GrPPD3, and GrZML6) increased. Particularly, 7 genes (GrJAZO4, GrJAZ05, GrJAZ08, GrJAZ12, GrJAZ13, GrJAZ15, and GrTIFY2) showed expression changes at abs $[\log 2$ (fold change) $>1$, indicating that these genes varied by greater than 2-fold in the expression level. Remarkably, dynamic and strong changes were observed in the expression of 2 genes, GrJAZ04 and GrJAZ12. During the secondary cell wall thickening stage, the expression level of 3 genes (GrJAZ07, GrJAZ09, and GrJAZ12) decreased, while that of 3 genes (GrJAZ08, GrJAZ13, and GrTIFY2) increased. During the fiber mature stage, the expression level of 2 genes (GrJAZO1 and GrTIFY2) always decreased, whereas that of 5 genes (GrJAZ05, GrJAZ09, GrPPD1, GrZML6, and GrZML8) always increased. The expression levels of 4 genes (GrJAZ03, GrJAZ04, GrJAZ08, and GrJAZ14) increased and then decreased, whereas that of 2 genes (GrZML2 and GrZML7) decreased and then increased.

Overall, most TIFY family genes showed development-dependent expression profiles in fiber cells, implicating these genes in various physiological processes during fiber growth. Additionally, 6 genes (GrJAZ01, GrJAZ04, GrJAZ08, GrJAZ09, GrTIFY2, and GrZML6) showed altered expression levels during all stages of fiber growth. Synthesizing RNA-seq expression profiles from 3 experiments showed that the transcriptional abundance of 3 genes (GrJAZ06, GrTIFY1, and GrZML1) were consistently comparatively low. Conversely, 3 genes (GrJAZ09, GrJAZ01, and GrJAZ08) were robustly transcribed and were ubiquitously and differentially expressed in all comparisons. Specifically, GrJAZ09 always showed the highest abundance among this family. In summary, most $T I F Y$ family genes played a role in both developmental transitions and fiber growth in cotton. Several genes (GrJAZ09, GrJAZ01, and GrJAZ08) should be further examined for future molecular design and fiber improvement.

\section{DISCUSSION}

Member of TIFY family are important TFs, and have been identified in many species. However, little information has been accumulated about this family in Gossypium. G. raimondii is an important diploid and wild species, whose ancestors contributed the D genome to the current main cultivated species by ancient interspecific hybridization. The release of $G$. raimondii genome sequences laid the foundation of comprehensive identification and characterization of the TIFY family in the important diploid species. Identification and analysis of the TIFY gene family in G. raimondii will provide valuable asset for the improvement of tetraploid cultivated species ( $G$. hirsutum and G. barbadense).

\section{Identification of the TIFY family genes}

In this study, we identified 28 TIFY genes in G. raimondii genome, among which 15 
belong to the $J A Z$ subfamily, 8 to the $Z M L$ subfamily, 3 to the $P P D$ subfamily, and 2 to the TIFY subfamily. Compared with other TFs identified in G. raimondii, such as bHLH (208 genes) and MYB (219 genes) (Wang et al., 2012a), the TIFY family is not a large family. Compared with other comprehensively surveyed plant TIFYs [34 TIFY family genes in soybean, 27 in maize, 25 in poplar, 20 in rice, 18 in Arabidopsis, and 15 (unpublished data) in cacao], the G. raimondii TIFY family ranks second with 28 phylogenetically expanded genes. Additionally, comparison with other plants indicated that the striking expansion and diversification of the TIFY family (28 copies), perhaps along with $3 P P D$ gene copies in G. raimondii, suggests that these TIFYs play crucial roles in the environmental adaptability and physiological maintenance of $G$. raimondii, which can survive in arid and stressful environments.

In order to absolutely identify all $T I F Y$ genes in G. raimondii, we also recur to other means. For example, after synteny detection based on the arrangement of genes on chromosome (dispersed segmental duplications), we conducted dc-megablast (database: G. raimondii genome; query: exons of TIFY family genes; e-value: $10^{-6}$ ) to explore the presence of evolving pseudo genes or relics in TIFYs. We found no other genomic regions encoding TIFY family members. Twenty-eight $T I F Y$ genes were also supported by transcript data from RNAsequencing. Thus, there were 28 members of $T I F Y$ family in G. raimondii.

\section{Evolution of the TIFY family genes}

Phylogenetic analysis and comparative genomic analyses are usually conducted to gain insight into evolutionary relationships of several genes or species. The phylogenetic tree of G. raimondii TIFY family genes indicated that the members of the JAZ subfamily were classified into several clades, agreeing with that in Arabidopsis (Vanholme et al., 2007), rice (Ye et al., 2009), and grape (Zhang et al., 2012), but the topology was not similar to those produced in these species. For example, the tree and the mean distances among subfamilies indicated that the relationship (distance, 5.487) of PPDs $v s$ ZMLs was much greater than that (4.505) of PPDs $v s$ some JAZs, or that (3.372) of ZMLs $v s$ other JAZs. However, the tree described by Zhang et al. (2012) indicated that $V v P P D$ s and $V v Z M L s$ clustered together in the grape, while the tree described by Vanholme et al. (2007) indicated that $A t Z M L$ s were not closely related to the collection of AtPPDs and AtJAZs, which clustered together in Arabidopsis. Therefore, the TIFY family in G. raimondii did not evolve in concert with that in other species. The tree of 6 species indicated that Gossypium was more closely related to the T. cacao genome (suggesting that they perform similar roles), which agreed with the results of Wang et al. (2012a), but did not show that Gossypium was closely related to the dicotyledonous Arabidopsis genome (Lin et al., 2010). Thus, inferring the role of the TIFY protein from the model plant Arabidopsis to Gossypium should be done with caution, although TIFYs in G. raimondii were mainly identified by homology. However, transferring the information between perennial WOODY species, such as G. raimondii and T. cacao, was possible.

From the perspectives of the primary structure of a peptide or protein, phylogenetic tree provided the information of evolutionary relationships. Additionally, from the arrangement of genes on chromosome, comparative genomic analyses (including intra-species and inter-species) can provide further insight into evolutionary relationships. Intra-species synteny analyses indicated that most TIFY genes were located in synteny regions, which implied that large-scale genomic duplication contributed to the expansion of the TIFY family. This kind of large-scale-duplication events had been undergone by many species in the plant kingdom 
(Paterson et al., 2012). Unneglectably, a few TIFY genes escaped from intra-species synteny blocks. The cases may result from the rearrangement and diversification following duplication (Wang et al., 2012a). Inter-species synteny analyses indicated that synteny regions containing $G$. raimondii TIFY family genes were also present in the comparison of $G$. raimondii vs $A$. thaliana or T. cacao, signifying that these genes or chromosome segment had common ancestors and play the similar and concerted roles in biological processes across species. Additionally, results of comparative genomic analyses are in agreement with that of phylogenetic analysis.

In summary, genome-wide identification and analysis of the TIFY family gene in $G$. raimondii has been carried out in this study. Twenty-eight $T I F Y$ genes are identified, which are diversity across clades of phylogenetic tree. The expansion of the TIFY family in G. raimondii may result from tandem and segmental duplication. Many of these genes may share common ancestors with that in other species, such as A. thaliana, T. cacao, and so on. RNA-seq and expression profiles indicated that most $T I F Y$ family genes were involved in fiber development. This study will provide very useful information for future fiber development.

\section{Conflicts of interest}

The authors declare no conflict of interest.

\section{ACKNOWLEDGMENTS}

Research supported by the National Natural Science Foundation of China (\#30971821), National Transgenic Plants Project of China (\#2011ZX08005-002), and the China Agriculture Research System (\#CARS-18-45). The sponsors of this study did not participate in study design, data collection or analysis, paper preparation, or the decision to publish the study.

\section{Supplementary material}

\section{REFERENCES}

Aparicio-Fabre R, Guillen G, Loredo M, Arellano J, et al. (2013). Common bean (Phaseolus vulgaris L.) PvTIFY orchestrates global changes in transcript profile response to jasmonate and phosphorus deficiency. BMC Plant Biol. 13: 26.

Bai YH, Meng YJ, Huang DL, Qi YH, et al. (2011). Origin and evolutionary analysis of the plant-specific TIFY transcription factor family. Genomics 98: 128-136.

Cannon SB, Mitra A, Baumgarten A, Young ND, et al. (2004). The roles of segmental and tandem gene duplication in the evolution of large gene families in Arabidopsis thaliana. BMC Plant Biol. 4: 10.

Chacón-López A, Ibarra-Laclette E, Sánchez-Calderón L, Gutiérrez-Alanís D, et al. (2011). Global expression pattern comparison between low phosphorus insensitive 4 and WT Arabidopsis reveals an important role of reactive oxygen species and jasmonic acid in the root tip response to phosphate starvation. Plant Signal Behav. 6: 382-392.

Chenna R, Sugawara H, Koike T, Lopez R, et al. (2003). Multiple sequence alignment with the Clustal series of programs. Nucleic Acids Res. 31: 3497-3500.

Chini A, Fonseca S, Chico JM, Fernández-Calvo P, et al. (2009). The ZIM domain mediates homo- and heteromeric interactions between Arabidopsis JAZ proteins. Plant J. 59: 77-87.

Chung HS, Niu YJ, Browse J and Howe GA (2009). Top hits in contemporary JAZ: an update on jasmonate signaling. Phytochemistry 70: 1547-1559.

Crooks GE, Hon G, Chandonia JM and Brenner SE (2004). WebLogo: A sequence logo generator. Genome Res. 14: 1188-1190.

Demianski AJ, Chung KM and Kunkel BN (2012). Analysis of Arabidopsis JAZ gene expression during Pseudomonas syringae pathogenesis. Mol. Plant Pathol. 13: 46-57. 
Flagel LE, Wendel JF and Udall JA (2012). Duplicate gene evolution, homoeologous recombination, and transcriptome characterization in allopolyploid cotton. BMC Genomics 13: 302.

Graves DA and Stewart JM (1988). Chronology of the differentiation of cotton (Gossypium hirsutum L) fiber cells. Planta 175: 254-258.

Grunewald W, Vanholme B, Pauwels L, Plovie E, et al. (2009). Expression of the Arabidopsis jasmonate signalling repressor JAZ1/TIFY10A is stimulated by auxin. Embo. Rep. 10: 923-928.

He DH, Lin ZX, Zhang XL, Nie YC, et al. (2007). QTL mapping for economic traits based on a dense genetic map of cotton with PCR-based markers using the interspecific cross of Gossypium hirsutum x Gossypium barbadense. Euphytica 153: 181-197.

Jiang CX, Wright RJ, El-Zik KM and Paterson AH (1998). Polyploid formation created unique avenues for response to selection in Gossypium (cotton). Proc. Natl. Acad. Sci. U. S. A. 95: 4419-4424.

Koonin EV (2005). Orthologs, paralogs, and evolutionary genomics. Annu. Rev. Genet. 39: 309-338.

Libault M, Joshi T, Benedito VA, Xu D, et al. (2009). Legume transcription factor genes: what makes legumes so special? Plant Physiol. 151: 991-1001.

Lin LF, Pierce GJ, Bowers JE, Estill JC, et al. (2010). A draft physical map of a D-genome cotton species (Gossypium raimondii). BMC Genomics 11: 395.

Lyons E, Pedersen B, Kane J, Alam M, et al. (2008). Finding and comparing syntenic regions among Arabidopsis and the Outgroups papaya, poplar, and grape: CoGe with Rosids. Plant Physiol. 148: 1772-1781.

Nishii A, Takemura M, Fujita H, Shikata M, et al. (2000). Characterization of a novel gene encoding a putative single zinc-finger protein, ZIM, expressed during the reproductive phase in Arabidopsis thaliana. Biosci. Biotech. Bioch. 64: 1402-1409.

Paterson AH, Wendel JF, Gundlach H, Guo H, et al. (2012). Repeated polyploidization of Gossypium genomes and the evolution of spinnable cotton fibres. Nature 492: 423-428.

Qi TC, Song SS, Ren QC, Wu DW, et al. (2011). The jasmonate-ZIM-domain proteins interact with the WD-repeat/bHLH/ MYB complexes to regulate jasmonate-mediated anthocyanin accumulation and trichome initiation in Arabidopsis thaliana. Plant Cell 23: 1795-1814.

Rambaldi D and Ciccarelli FD (2009). FancyGene: dynamic visualization of gene structures and protein domain architectures on genomic loci. Bioinformatics 25: 2281-2282.

Rice P, Longden I and Bleasby A (2000). EMBOSS: The European molecular biology open software suite. Trends Genet. 16: 276-277.

Seo JS, Joo J, Kim MJ, Kim YK, et al. (2011). OsbHLH148, a basic helix-loop-helix protein, interacts with OsJAZ proteins in a jasmonate signaling pathway leading to drought tolerance in rice. Plant J. 65: 907-921.

Shikata M, Matsuda Y, Ando K, Nishii A, et al. (2004). Characterization of Arabidopsis ZIM, a member of a novel plantspecific GATA factor gene family. J. Exp. Bot. 55: 631-639.

Shiu SH and Bleecker AB (2003). Expansion of the receptor-like kinase/Pelle gene family and receptor-like proteins in Arabidopsis. Plant Physiol. 132: 530-543.

Staswick PE (2008). JAZing up jasmonate signaling. Trends Plant Sci. 13: 66-71.

Tamura K, Peterson D, Peterson N, Stecher G, et al. (2011). MEGA5: Molecular evolutionary genetics analysis using maximum likelihood, evolutionary distance, and maximum parsimony methods. Mol. Biol. Evol. 28: 2731-2739.

Tang HB, Bowers JE, Wang XY, Ming R, et al. (2008). Synteny and collinearity in plant genomes. Science 320: 486-488.

Thines B, Katsir L, Melotto M, Niu Y, et al. (2007). JAZ repressor proteins are targets of the SCFCO11 complex during jasmonate signalling. Nature 448: 661-665.

Trapnell C, Roberts A, Goff L, Pertea G, et al. (2012). Differential gene and transcript expression analysis of RNA-seq experiments with TopHat and Cufflinks. Nat. Protoc. 7: 562-578.

Vanholme B, Grunewald W, Bateman A, Kohchi T, et al. (2007). The tify family previously known as ZIM. Trends Plant Sci. 12: 239-244.

Wan Q, Zhang H, Ye W, Wu H, et al. (2014). Genome-wide transcriptome profiling revealed cotton fuzz fiber development having a similar molecular model as Arabidopsis trichome. PLos One 9: e97313.

Wang KB, Wang ZW, Li FG, Ye WW, et al. (2012a). The draft genome of a diploid cotton Gossypium raimondii. Nat. Genet. 44: 1098-1103.

Wang YP, Tang HB, DeBarry JD, Tan X, et al. (2012b). MCScanX: a toolkit for detection and evolutionary analysis of gene synteny and collinearity. Nucleic Acids Res. 40: e49.

Wasternack C (2007). Jasmonates: An update on biosynthesis, signal transduction and action in plant stress response, growth and development. Ann. Bot. 100: 681-697.

White DWR (2006). PEAPOD regulates lamina size and curvature in Arabidopsis. Proc. Natl. Acad. Sci. U.S.A. 103: 1323813243. 
Wray GA, Hahn MW, Abouheif E, Balhoff JP, et al. (2003). The evolution of transcriptional regulation in eukaryotes. Mol. Biol. Evol. 20: 1377-1419.

Ye HY, Du H, Tang N, Li XH, et al. (2009). Identification and expression profiling analysis of TIFY family genes involved in stress and phytohormone responses in rice. Plant Mol. Biol. 71: 291-305.

Zhang YC, Gao M, Singer SD, Fei ZJ, et al. (2012). Genome-wide identification and analysis of the TIFY gene family in grape. PLos One 7: e44465.

Zhu D, Bai X, Luo X, Chen Q, et al. (2013). Identification of wild soybean (Glycine soja) TIFY family genes and their expression profiling analysis under bicarbonate stress. Plant Cell Rep. 32: 263-272. 